\title{
A comparative study of verbal IQ, performance IQ and verbal IQ- performance IQ disparity among Turner syndrome patients and patients with primary amenorrhoea due to other aetiologies
}

\author{
Lakshmi R. Lakshman $^{1}{ }^{*}$, Rahul Lakshman ${ }^{1}$, D. M. Vasudevan ${ }^{2}$
}

\author{
${ }^{1}$ Department of Obstetrics \& Gynecology, Amrita Institute of Medical Sciences, Kochi-682041, India \\ ${ }^{2}$ Department of Biochemistry, Amrita Institute of Medical Sciences, Kochi-682041, India
}

Received: 29 November 2012

Accepted: 11 December 2012

*Correspondence:

Dr. Lakshmi R. Lakshman,

E-mail: nanolakshmi@gmail.com

\begin{abstract}
Background: Assessing disparity in IQ is important in selecting an occupation and thus helping people lead a productive life. In Turner syndrome patients, this shall be more important as assessment of verbal IQ- and performance IQ disparity could be used in helping them select an occupation so that productivity and quality of life is not grossly compromised.

Methods: Based on karyotyping, 30 patients with turner syndrome and 30 patients with primary amenorrhoea due to other aetiologies were selected for the study. Cytogenetic analysis was done for every case using G-banding technique. Assessment of intellectual functions was done using Wechsler Adult Intelligence Scale (WAIS).

Results: Mean value of PIQ of X0 (turner) was 74.67 and that of XX (other amenorrhoea patients) was 90.30. Mean value of VIQ of X0 (turner) was 93.67 and that of XX (other amenorrhoea patients) was 93.60. Mean value of VIQ and PIQ disparity of X0 (turner) was 18.67 and that of XX (other amenorrhoea patients) was 3 .

Conclusions: Turner syndrome patients have high VIQ-PIQ disparity when compared to other primary amenorrhoea patients.
\end{abstract}

Keywords: Amenorrhoea, Turner syndrome, Verbal IQ (VIQ), Performance IQ (PIQ), karyotype, Wechsler Adult Intelligence Scale (WAIS)

\section{INTRODUCTION}

Turner syndrome is a genetic abnormality caused by the absence of one sex chromosome. Normal female chromosome complement is depicted as 46XX. This means that normal females have 44 somatic chromosomes and 2 sex chromosomes $(44+2=46)$. Females with Turner syndrome have 44 somatic chromosomes and a single sex chromosome $(44+1=45)$. Their chromosome complement is depicted as ' $45 \mathrm{X} 0$ '. Turner recognized the combination of sexual infantilism, webbed neck and cubitus valgus as a distinct entity. ${ }^{1}$ Ford et al showed that females with Turner syndrome were missing one sex chromosome. ${ }^{2}$

Turner syndrome consists of short stature, webbed neck, shield chest, hypoplastic nails, short metacarpals, coarctation of aorta, streak gonads, peripheral lymphoedema at birth and multiple pigmented nevi. Most of the females with turner syndrome are infertile and do not spontaneously menstruate or develop secondary sexual characteristics. The prevalence is $1 / 2500$ live female births. Approximately $98-99 \%$ of Turner syndrome fetuses are spontaneously aborted. About onethird of Turner syndrome patients are diagnosed in the new born period, one-third during childhood and onethird during the teenage years when failure to go through puberty becomes evident. A minimal diagnostic criterion is an abnormal karyotype in which all or part of one of the $\mathrm{X}$ chromosomes is absent. Clinical diagnosis is based on the overall pattern of anomalies.

A karyotype is the characteristic chromosome complement of a eukaryotic species. The preparation and study of karyotype is part of cytogenetics. In normal diploid organisms, autosomal chromosomes are present 
in two copies. The study of whole sets of chromosomes is known as karyology. The chromosomes are depicted by rearranging a micro photograph in a standard format known as a karyogram in pairs placed in order of size and position of centromere for chromosomes of the same size. Karyotyping can be used to studying chromosomal aberrations and could be instrumental in prevention of abnormal births. It can also be used for assessing cellular function, taxonomic relationships and to gather information about past evolutionary events. Normal human karyotype contains 22 pairs of autosomal chromosomes and one pair of sex chromosomes. Normal male karyotype is denoted as 46XY and normal female karyotype is denoted as $46 \mathrm{XX}$.

Primary amenorrhoea is defined as the absence of menstruation by 16 years of age in the presence of normal secondary sexual characteristics or by 14 years of age when there is no visible secondary sexual characteristics. There are many causes for primary amenorrhoea. An important genetic cause for primary amenorrhoea is gonadal dysgenesis. The reason for which karyotyping should be done in primary amenorrhoea is to differentiate between Turner syndrome and other causes for gonadal dysgenesis. In case of XO-XY or XX-XY mosaicism, which can also cause primary amenorrhoea, there is high chance for developing gonadoblastoma (ovarian cancer) and heterosexual development. Early detection by karyotyping and prophylactic gonadectomy done there after can prevent ovarian cancer.

Turner syndrome patients have some learning disabilities, particularly with regard to spatial perception, visualmotor co-ordination and mathematics. Most of them are not mentally retarded. Earlier studies on Turner syndrome had methodological short comings, including faulty reporting of mental retardation in some patients when in fact, reduced performance IQ in the presence of a normal verbal IQ results from specific deficits in spatial ability rather than global reduction in intelligence. Visual-spatial deficits have been related to reduce functioning in the right cerebral hemisphere, especially the right parietal lobe. Linden MG -noted that features such as low speech development, hyperactivity, learning disabilities and short stature could contribute to poor self-image and low self-esteem during adolescence in Turner syndrome patients. ${ }^{3}$ Romans S and Ross JL found problems in spatial/perceptual skills, visual-motor integration, affect recognition, visual memory and attentional abilities. ${ }^{4,5}$ They were found to have average language skills.,

Assessing disparity in IQ is very interesting and important in selecting an occupation and thus helping people lead a productive life. In Turner syndrome patients, this shall be more important as assessment of verbal IQ- and performance IQ disparity could be used in helping them select an occupation so that productivity and quality of life is not grossly compromised. Although some studies were conducted abroad, the present study among the Turner syndrome patients is first of its kind in
Kerala. Even though Turner syndrome is not a fatal condition, the psychological and social significance of the condition is very high. The extremely low prevalence of Turner syndrome makes this study rare, unique and important. Turner syndrome patients are not necessarily mentally retarded, but some studies conducted elsewhere found that the IQ disparity (verbal IQ / performance IQ) exists so as performance IQ is low. This was done among western population, where the education system is entirely different. In India, general educational practices are verbal IQ oriented rather than performance IQ oriented. There is not much studies related to the IQ of girls with Turner syndrome in India. Studying the IQ disparity among the Turner syndrome patients in the context of the cultural, social, psychological and educational background of Kerala is what the investigator attempts through this study. Conducting a study among the Turner syndrome population in Kerala could be used in helping them in selecting the type of education and occupation best suited for them. For a patient with lower performance IQ compared to the verbal IQ, the occupational practices should be more verbal IQ oriented. This has not been studied among the Turner syndrome girls in Kerala, where the literacy rate and educational standards are high. By conducting a comparative study of the IQ disparity between Turner syndrome patients and patients with primary amenorrhoea due to other aetiologies, will help to understand the Kerala scenario of the problem under study. Through karyotyping, one might detect XO-XY or XX-XY mosaicism, which can also cause primary amenorrhoea. In patients with mosaicism, there is high chance for developing gonadoblastoma (ovarian cancer) and heterosexual development. Early detection by karyotyping and prophylactic gonadectomy done there after can prevent ovarian cancer.

\section{METHODS}

A total of 156 patients with primary amenorrhoea who attended either in endocrinology or gynecology outpatient department at Amrita Institute of Medical Science were consulted in detail regarding the importance of karyotyping in the evaluation of primary amenorrhoea. The role of karyotyping in early detection of risk of gonadoblastoma (gonadal cancer) was also explained and stressed upon. All 156 patients were willing to do the karyotyping. Based on karyotyping, 30 patients with turner syndrome and 30 patients with primary amenorrhoea due to other aetiologies were selected for the study. The permission from the institutional ethics committee was obtained before starting the study. The personal data sheet was designed to collect information regarding the socio-demographic aspects, illness and treatment related variables of the participants. Full clinical examination was done for each case. Thorough investigation included pelvic sonography.

Cytogenetic analysis was done for every case using Gbanding technique. ${ }^{6,7}$ Karyotyping nomenclature was 
according to the international system for human cytogenetic nomenclatures (ISCN). ${ }^{8}$

Assessment of intellectual functions was done using Wechsler Adult Intelligence Scale (WAIS). ${ }^{9}$ This scale comprised subtests for verbal and performance intelligence. Scoring and scaling of each test was done, verbal intelligence quotient (VIQ) and performance intelligence quotient (PIQ) were derived. Mean and standard deviation were calculated. Student's t-test was used to find out the significant difference if any, between variables.

\section{RESULTS}

Mean value of PIQ of X0 (turner) was 74.67 and that of XX (other amenorrhoea patients) was 90.30. From this mean value it is clear that turner syndrome patients have low PIQ when compared to other primary amenorrhoea patients (Table 1).

Table 1: Performance IQ (PIQ) of Turner syndrome patients and patients with primary amenorrhoea due to other aetiologies.

\begin{tabular}{|llllll|}
\hline Sr. No. & Karyotype & $n$ & Mean & SD & t \\
\cline { 1 - 5 } 1 & $\mathrm{x} 0$ & 30 & 74.67 & 8.984 & \multirow{2}{*}{$5.64 * *$} \\
\hline 2 & $\mathrm{xx}$ & 30 & 90.30 & 12.237 & \\
\hline \multicolumn{5}{c}{$* *$ significant at 0.01 level } \\
\hline
\end{tabular}

Table 2: Verbal IQ (VIQ) of Turner syndrome patients and patients with primary amenorrhoea due to other aetiologies.

\begin{tabular}{|llllll|}
\hline Sr. No. & Karyotype & $n$ & Mean & SD & t \\
\cline { 1 - 5 } 1 & $\mathrm{x} 0$ & 30 & 93.67 & 7.411 & \multirow{2}{*}{0.02} \\
\hline 2 & $\mathrm{xx}$ & 30 & 93.60 & 13.449 & \\
\hline
\end{tabular}

Table 3: Disparity between Verbal IQ (VIQ) and Performance IQ (PIQ) in patients with Turner syndrome and primary amenorrhoea due to other aetiologies.

\begin{tabular}{|llllll|}
\hline Sr. No. & Karyotype & $n$ & Mean & SD & t \\
\cline { 1 - 4 } 1 & $\mathrm{x} 0$ & 30 & 18.67 & 4.971 & \multirow{2}{*}{$10.51 * *$} \\
\hline 2 & $\mathrm{xx}$ & 30 & 3.00 & 6.475 & \\
\hline \multicolumn{5}{c}{$* *$ significant at 0.01 level } \\
\end{tabular}

Mean value of VIQ of X0 (turner) was 93.67 and that of XX (other amenorrhoea patients) was 93.60 (Table 2). Hence, there is no difference in VIQ between turner syndrome patients and patients with other primary amenorrhoea.

Mean value of VIQ and PIQ disparity of X0 (turner) was 18.67 and that of XX (other amenorrhoea patients) was 3 (Table 3). From the mean value it is clear that Turner syndrome patients have high VIQ-PIQ disparity when compared to other primary amenorrhoea patients.

\section{DISCUSSION}

There is a significant difference in performance IQ between Turner syndrome patients and other primary amenorrhoea patients. In Turner syndrome, performance IQ is lower when compared to other primary amenorrhoea patients. But there is no significant difference in verbal IQ. Verbal IQ-Performance IQ disparity is significant between Turner syndrome patients and other primary amenorrhoea patients.

Wechsler stated that verbal IQ-performance IQ score disparity of 15 points is important for saying that there is a verbal IQ-performance IQ disparity. ${ }^{9}$ Here in this study there is a mean disparity of 18.67. Cronbach stated that verbal IQ-performance IQ disparity score has an estimated reliability ( 0.74 at the $99 \%$ level of confidence) high enough for justify drawing conclusion about the person whose verbal IQ-performance IQ disparity is 15 points or so. ${ }^{10}$ Kaufman stated that verbal IQperformance IQ disparity of 15 or more should caution the clinician to use as a statistical abnormality criterion for diagnostic or educational purpose. ${ }^{11}$ In our study we have a mean VIQ-PIQ disparity of 18.67 with a 
significance of t-value 10.51 with $99 \%$ confidence, which prove that the VIQ-PIQ disparity in our population is significant clinically for diagnosis, educational counseling and occupational guidance, so as to lead a socially productive life for those individuals with Turner syndrome.

Individuals with Turner syndrome have selective impairment in nonverbal, visual-spatial processing and as a group score lower on the performance than on the verbal subsection of standardized intelligence tests. ${ }^{12}$ The specific neuropsychological deficits that may affect adaptation include four interacting areas of functioning: visual-spatial organization deficits (e.g. difficulty in driving), defects in social cognition (e.g. failure to appreciate subtle social cues), problems with nonverbal problem solving (e.g. mathematics), and psychomotor deficits (e.g. clumsiness). It is possible that some of these neuropsychological deficits may be improved by estrogen therapy. 5

Findings of temporal lobe differences (disproportionately reduced right hippocampal volume and larger left amygdala grey matter) may relate to the memory and social cognitive deficits noted in Turner's syndrome. Research using fMRI documents parietal lobe and prefrontal cortex differences. Consistent with visual motor problems. There is evidence to suggest a chromosomally determined process of neuro-cognitive development, which, in turn, affects cognitive, social, behavioural development in individuals with Turner's syndrome. Skusse and colleagues explored one possible pathway by which neurocognitive deficits could affect social function, in series of investigations of the inability of Turner syndrome patients to accurately interpret facial affect. $^{13,14}$ This cognitive inability to read and respond effectively to facial affective cues may then result in social and behavioural deficits. ${ }^{13,14}$ Our study also proved the verbal IQ-performance IQ disparity in turner syndrome is significant. So we suggest strategies to enhance social and behavioural development include: individual testing of cognitive abilities at the onset of school with on-going attention to the need for additional assessment and tutoring to keep up with grade level expectations. Coaching to assure active social involvement especially during the middle and high school year. Preparing for emancipation and successful transition into the working environment with ageappropriate expectation for participation chores, social activities and jobs outside the home. Education planning before high school so that girls who have significant learning problems can be appropriately guided including how to qualify for examinations needed at college level.

\section{REFERENCES}

1. Turner HH. A syndrome of infantilism, congenital webbed neck, and cubitus valgus. Endocrinology 1938;23:566-74.

2. Ford CE, Jones KW, Polani PE, De Almeida JC, Briggs JH. A sex-chromosome anomaly in a case of gonadal dysgenesis (Turner's syndrome). Lancet 1959;1(7075):711-3.

3. Linden MG, Bender BG, Harmon RJ, Mrazek DA, Robinson A. 47,XXX: what is the prognosis? Pediatrics 1988;82:619-30.

4. Romans $\mathrm{S}$, et al. Executive function in girls with Turner's syndrome. Dev Neuropsychol 1997;132340.

5. Ross JL, et al. Effects of estrogen on nonverbal processing speed and motor function in girls with Turner's syndrome. J Clin Endocrinol Metab 1998;83:3198-204.

6. Verma RS and Babu A. Human chromosomes: Principles and techniques. Tissue culture techniques and chromosome preparation. In human chromosome manual of basic techniques RS Verma, A. Babu (eds) 2nd Edition. Pergamon Press, McGraw-Hill, New York;1995.

7. Pinkle D, Gray J, Trask B., Van den Engh G, Fuscoe J. and Van Dekken H. Cytogenetic analysis by in situ hybridization with fluorescently labeled nucleic acid probes. Cold Spring Harbor Symp. Quaut Biol 1986;51: 151-7.

8. ISCN: An international system for human Cytogenetic nomenclature. Mitelmen F (ed), S Karger, Basel, New York, 1995.

9. Wechsler D. Manual of Wechsler Adult Intelligence Scale (WAIS). Psychological Corporation, New York;1996.

10. Cronbach LJ. Essentials of Psychological Testing. New York: Harper \& Brothers; 1961.

11. Kaufman AS. Tests of intelligence. In Sternberg RJ (Ed.), Handbook of intelligence. New York: Cambridge University Press, 2000;445-76.

12. Ross JL, McCauley E, Roeltgen D, Long L, Kushner H, Feuillian P, et al. Self-concept and behavior in adolescent girls with Turner syndrome: Potential estrogen effects. J Clin Endocrinol Metab 1996;81:926-31.

13. Lawrence $\mathrm{K}$, et al. Face and emotion recognition deficits in Turner syndrome: a possible role for $\mathrm{X}$ linked genes in amygdala development. Neuropsychology 2003;17:39-49.

14. Lawrence $\mathrm{K}$, et al. Interpreting gaze in Turner syndrome: impaired sensitivity to intention and emotion, but preservation of social cueing. Neuropsychologia 2003;41:894-905.

DOI: $10.5455 / 2320-1770$. ijrcog001212

Cite this article as: Lakshman LR, Lakshman R, Vasudevan DM. A comparative study of verbal IQ, performance IQ and verbal IQperformance IQ disparity among Turner syndrome patients and patients with primary amenorrhoea due to other aetiologies. Int J Reprod Contracept Obstet Gynecol 2012;1:22-5. 